\title{
Perspective Crisis in Pain Management
}

\author{
${ }^{*}$ Edward H Tobe
}

Clinical Professor, Department of Psychiatry, Cooper Medical School of Rowan University, USA

Received: April 10, 2017; Accepted:April 25, 2017; Published: May 12, 2017

*Corresponding author: Edward H Tobe, 1001 Lincoln Drive West Suite B, Marlton, NJ 08053-1534, USA, Tel: 856-983-4940; E-mail: Edward.tobe@comcast.net

\begin{abstract}
Throughout the United States, various authorities have expressed alarm about opiate abuse. The subject is like a river with many tributaries. Perhaps there has been an excessive focus on one tributary, the prescribing habits of physicians. This focus may impair the physician's capacity to empathize with the patient's pain.

Pain is a disease of the central nervous system, not merely a complaint. Currently there are automatic algorithms to treat pain that lack careful evaluation of the individual person. The person is fitted into the algorithm not the algorithm fits the patient. This author encourages an algorithm based upon a comprehensive evaluation of the individual's perceptions, vocational and avocational interests, and family life.
\end{abstract}

\section{Limitations of comments}

One physician's observations of patient care are limited. Other physicians will perhaps read this perspective and share their opinion.

Keywords: Opiate crisis; Pain; Pain management

\section{Pain reception and brain}

Signaling danger to the body, pain enables survival. Pain sensitivity varies individually. Two major types of fibers transmit pain: A and C. Type A fibers are myelinated and faster than the unmyelinated C fibers. The cell body for the primary neuron is in the dorsal root ganglion. Chronic pain as well as acute pain reaches brain through either the Trigeminothalamic tract to the ventral posterior medial thalamic nucleus or the spinothalamic tract to the ventral posterior lateral thalamic nucleus. Pain reception is a peripheral to central nervous system biological process. Chronic pain is a biological process that maintains vigilance as if the body were at risk. Unlike acute pain, chronic pain may change cellular morphology and neuronal connectivity; when pain remits, there may be recovery in part or whole of neuroplasticity $[1,2]$. Examples of areas of altered neuroplasticity include the anterior cingulate cortex, insular cortex and operculum, dorsolateral prefrontal cortex, orbitofrontal cortex and right thalamus $[1,2]$. The degree of cortical gray matter loss was associated with length of pain and was more severe in neuropathic pain. In one study chronic pain patients showed 5 to $11 \%$ less neocortical gray matter than controls. The amount of loss was equivalent to 10 to 20 years of normal aging. Chronic pain is no longer a symptom but represents a change in the CNS to a maladaptive state that is a disease of the CNS [1].

\section{Opiates help and harm}

The United States Centers for Disease Control (CDC) sounded alarm about the association of prescription opioids to morbidity and mortality. Opioids are derived from opium poppy or synthetic analogues [3]. Opioids have a significant potential to induce craving, tolerance, physiological and psychological dependence. Death results from impaired breathing. The CDC has suggested that a combination of increased prescriptions of opiates for nonfatal pain and the increased availability of heroin has created a crisis of deaths associated to prescription opiates and heroin.

People living with chronic pain often suffer comorbid depression. The combination of depression and chronic pain is associated with an increased rate of suicide."The true numbers of failed attempts and successful suicides are unknown and may never be determined. Yet, risk factors for suicidal ideation are so high in this population that it must be assumed that some proportion of those who die of drug overdoses might have intended to end their lives, not just temporarily relieve their pain [4]."

For people suffering chronic pain, treatment addresses quality of life. Chronic non-fatal pain is associated with a lessened or lost ability to exercise, experience normal sleep, perform household duties, attend social/religious activities, and enjoy sexual relations. Frequently relationships with family or friends are compromised. There are moral concerns about not providing adequate pain relief to allow a better quality of life regardless of the cause of pain. (5)

\section{Pain Management and social issues}

Some researchers, clinicians and politicians have joined the movement to discourage the prescription of opiates. Opinions derive from well meaning to vested financial or political interests. Chronic pain patients fear abandonment.

Patients and their families want symptom reduction and improvement in their quality of life. Third-party payers are private or government payers with vested interest to control expenditures. Pharmaceutical corporations have found the 
sustained oral opiate release products lucrative. The weighing of variables to determine pain management outcome depends upon whom is asked, patients or payer versus independent healthcare providers.

\section{Treatment algorithm}

Prudence requires knowing the patient's past, appreciating the complexities of the patient's present to enable an educated inference about their future. Myopic treatment strategies focus on pain relief through lucrative surgical and invasive procedures without identifying risks. The physician successfully performs procedures; however, because the procedure(s) did not enable the patient to reengage in vocational and avocational activities; the patient perceives a failed treatment. Animosity may develop because physician and patient perceive different outcomes.

Opiates are prescribed with a "pain contract" to protect the prescriber. The use of a visual or number scale to determine the person's level of pain encourages patients to focus on a pain scale as if reporting a glucose level. The above treatment algorithm encourages dependence on procedures and pain suppressants.

Often due to a failure to obtain adequate information, during chronic pain management with opiates, patients vulnerable to cravings for CDS may use pain medicines excessively. When a routine urine screen shows misuse of the prescribed medications or the presence of medicine not prescribed, the patient may face precipitous termination from CDS pain management and put in jeopardy of withdrawal and/or illegally obtaining CDS. The patient often had a history of substance abuse that was ignored by the treating medical personnel. The practical realities of need to control pain often lead to purchasing the denied medicine from illegal sources, usually a very expensive endeavor. Heroin is much cheaper and becomes the source of pain management first by snorting and then administered intravenously. The patient has become a criminal.

\section{Physical Therapy (PT)}

Many PT centers are like a warehouse. The patient enters a room filled with many patients and a few physical therapy assistants. The patient receives a list of activities, told "no pain, no gain", provided ice or heat and scheduled for the next session. There is no individual guidance to ensure correct exercise movement of injured body parts. Instead of recognizing increased pain as a signal that the therapy needs reevaluated, the patient cooperates and becomes injured and may require surgery.

PT is critical to provide as much individual attention as needed to be certain of patient safety and effectiveness of treatment. Often overlooked is deep warm water aqua therapy. This therapy suspends the patient in warm water to permit full range of motion without body weight trauma.

\section{Often Ignored}

The evaluation of diet and nutrition helps prevent weight gain and ensures adequate nutrition for recovery.
Because of frustration, some people turn to carbohydrate craving and become obese. They face the complexities of obesity such as obstructive sleep apnea.

The ongoing assessment of mental state permits knowing the perceptions of the patient. This knowledge removes them from a conveyor belt and allows fine tuning of treatment.

A prolific literature demonstrates the bilateral relationship between chronic pain, psychiatric disorders, and suicide. Chronic pain patients often perceive psychiatric or psychological treatment as a denial of their suffering 'real' pain 'that is not just in my head' [5].

Either due to genetic proclivities or the pathophysiology of altered neuroplasticity from an existing psychiatric disease, patients with premorbid psychiatric disorders can be more vulnerable to mental deterioration especially with chronic pain. Medicine has long recognized the risk of aggravation of latent, asymptomatic disease or clinically evident disease from either psychological or physiological trauma.

Various classes of medicine that are not Controlled Dangerous Substances have been used to treat chronic pain. From this physician's experience, the most significant gap in the effective treatment of chronic pain is antidepressants. Antidepressant treatment of chronic pain has been helpful for over 50 years. Most experience has been with the TriCyclic Antidepressants (TCAs). Also highly effective are the underused Mono Amine Oxidase Inhibitors (MAOIs). In the absence of overt depression, antidepressants improve sleep, regulate mood, and modulate pain. Antidepressants usually do not provide complete pain relief. Realistically, patients in pain management prescribed high doses of opiates still complain of pain. Adverse events from TCAs or MAOIs are acceptable in a risk benefit analysis especially compared to the conveyor belt algorithm of invasive strategies and/or chronic opiate treatment. A response from antidepressant treatment requires adequate therapeutic doses provided over an adequate trial period.

Certain antidepressant drugs and antiepileptics are well marketed for pain control. The physician needs to look beyond commercials and industry supported studies. In this physician's experience, marketing and clinical effectiveness are not correlated.

\section{Summary}

This commentary does not oppose chronic opiate treatment that enhances quality-of-life; rather, the intent is to enhance individualization of treatment algorithms. The following suggestions may be prudent.

-Detail the patient's life history for diagnosed or undiagnosed drug or alcohol abuse. These patients present a risk of reawakening CDS craving.

-Psychiatric disorders whether diagnosed, denied, or undiagnosed present an increased risk of a compromised response to illness.

-Evaluate the effectiveness and expertise of provided physical 
therapy.

-Before instituting chronic opiate pain management, evaluate other options.

\section{References}

1. Apkarian AV, Sosa Y, Sonty S, Levy RM, Harden RN, Parrish TB, et al. Chronic back pain is associated with decreased prefrontal and thalamic gray matter density. J Neurosci. 2004;24(46):10410-10415.

2. Rodriguez-Raecke R, Niemeier A, Ihle K, Ruether W, May A. Structural brain changes in chronic pain reflect probably neither damage nor atrophy. PLoS One. 2013;8(2):e54475. doi:10.1371/journal. pone. 0054475

3. Paulozzi LJ, Ryan GW. Opioid analgesics and rates of fatal drug poisoning in the United States. Am J Prev Med. 2006;31(6):506-511.

4. Cheatle MD. Depression, chronic pain, and suicide by overdose: on the edge. Pain Med. 2011;12(Suppl 2):S43-S48. doi:10.1111/j.15264637.2011.01131.x

5. Radat F, Koleck M. [Pain and depression: cognitive and behavioural mediators of a frequent association]. Encephale. 2011;37(3):172-179. doi:10.1016/j.encep.2010.08.013 